\title{
Topology of knot spaces in dimension 3
}

\author{
RYAN BUDNEY \\ Mathematics and Statistics, University of Victoria \\ PO BOX 3045 STN CSC, Victoria, B.C., Canada V8W 3P4 \\ Email: rybu@uvic.ca
}

\begin{abstract}
This paper gives a detailed description of the homotopy type of $\mathcal{K}$, the space of long knots in $\mathbb{R}^{3}$, the same space of knots studied by Vassiliev via singularity theory. Each component of $\mathcal{K}$ corresponds to an isotopy class of long knot, and we list the components via the companionship trees associated to knots. The knots with the shortest companionship trees are: the unknot, torus knots, and hyperbolic knots. The homotopy type of these components of $\mathcal{K}$ were computed by Hatcher. In the case the companionship tree has more than one vertex, we give a fibre-bundle description of the corresponding components of $\mathcal{K}$, recursively, in terms of the homotopy types of components of $\mathcal{K}$ corresponding to knots with shorter companionship trees. The primary case studied in this paper is the case of a knot which has a hyperbolic manifold contained in the JSJ-decomposition of its complement. Moreover, the homotopy type of $\mathcal{K}$ as an $\mathrm{SO}_{2}$-space is determined, which gives a detailed description of the homotopy-type of the space of embeddings of $S^{1}$ in $S^{3}$.
\end{abstract}

AMS Classification numbers Primary: 57R40

Secondary: 57M25, 57M50, 57P48, 57R50

Keywords: spaces of knots, little cubes operad, embeddings, diffeomorphisms, JSJ 


\section{Introduction}

Let $\mathcal{K}$ denote the space of smooth embeddings of $\mathbb{R}$ in $\mathbb{R}^{3}$ which agree with a fixed linear embedding outside of $[-1,1] . \mathcal{K}$ is called the space of long knots in $\mathbb{R}^{3}$. The fixed linear embedding will be called the 'long axis.' To give a proper statement of the results in this paper, some familiarity with the JSJ-decomposition of 3-manifolds applied to knot complements in $S^{3}$ as in the paper [4] will be assumed. A very brief summary which fixes some notation is included in the following four paragraphs.

Given a long knot $f \in \mathcal{K}$ the JSJ-decomposition of the knot complement $C$ consists of a collection of incompressible tori $T \subset C$. To be precise, consider $S^{3}$ to be the one-point compactification of $\mathbb{R}^{3}$, thus $f$ has a one-point compactification which is an embedding of $S^{1}$ in $S^{3}$. $C$ is the complement of an open tubular neighbourhood of this embedded $S^{1}$ in $S^{3}$. Let $C \mid T$ denote the complement of an open tubular neighbourhood of $T$ in $C$. Form a graph $G_{f}$ whose vertices are the path-components of $C \mid T$, and whose edges are path-components of $T$. This graph has the structure of a rooted tree, with root $V$ given by the component of $C \mid T$ containing $\partial C$. Call this tree the JSJ-tree of $f$. The root manifold $V$ has the interesting property that it has a canonical re-embedding in $S^{3}$ as the complement of a Knot Generating Link (KGL) - this is a link $L=\left(L_{0}, L_{1}, \cdots, L_{n}\right)$ whose complement is prime and either Seifert fibred or atoroidal, such that the $n$-component sub-link $\left(L_{1}, L_{2}, \cdots, L_{n}\right)$ is the unlink. $n$ is the number of children of $V$ in the JSJ-tree. Moreover, $\overline{C \backslash V}=C_{J_{1}} \sqcup \cdots \sqcup C_{J_{n}}$ is the disjoint union of $n$ non-trivial knot complements. Thus, the knot $f$ is can be represented as a 'spliced' knot, $f=J \bowtie L$ where $J=\left(J_{1}, \cdots, J_{n}\right)$ is an $n$-tuple of non-trivial long knots whose complements are $C_{J_{1}}, \cdots, C_{J_{n}}$. One obtains the spliced knot $J \bowtie L$ from $L_{0}$ by a generalized satellite construction: fix $D=\left(D_{1}, \cdots, D_{n}\right)$ a collection of disjoint discs in $S^{3}$ such that $\partial D=\left(\partial D_{1}, \cdots, \partial D_{n}\right)=\left(L_{1}, \cdots, L_{n}\right)$, and fix $\nu D$ a closed tubular neighbourhood of $D . J \bowtie L$ is the union of $L_{0} \backslash \nu D$ together with the (untwisted) re-embedding of $L_{0} \cap \nu D_{i}$ along the knots $J_{i}$ for all $i \in\{1,2, \cdots, n\}$. Specifically, re-embed a tubular neighbourhood of $D_{i}$ using $J_{i}$ as the 'pattern,' like in the diagram below.
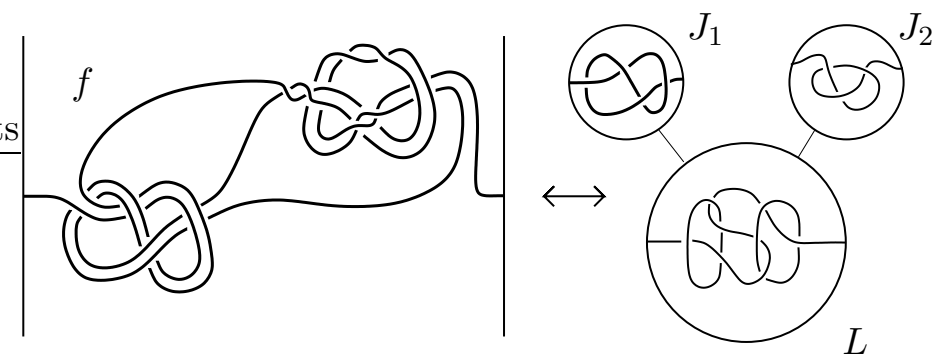

$f=J \bowtie L$ where $L$ is the Borromean rings

When $n=0$, interpret $J \bowtie L$ to be $L$. In this case $L$ is either a torus knot, a hyperbolic knot or an unknot. In the general case, after one writes $f$ as a splice, $f=J \bowtie L$, one can continue and write the knots of $J$ as spliced knots, iteratively. This process terminates, at which point the iterated splice construction for $f$ is precisely the JSJ-tree of $C$, with every vertex decorated by the appropriate KGL. This decorated tree $\mathbb{G}_{f}$ is called the companionship tree of $f$. $\mathbb{G}_{f}$ is a complete isotopy invariant of long knots. For the purpose of this paper, companionship trees will be used to index the components of $\mathcal{K}$. 
Seifert-fibred KGLs were classified by Burde and Murasugi. Thurston proved that non-Seifert fibred KGLs are hyperbolic. Kanenobu proved that hyperbolic KGLs exist with arbitrarily many components, thus any abstract finite rooted tree can be realized as the JSJ-tree $G_{f}$ of some long knot $f$. See [4] for complete references to all the above comments regarding JSJ-decompositions and companionship trees.

Given a knot $f \in \mathcal{K}$, label the path-component of $\mathcal{K}$ containing $f$ by $\mathcal{K}_{f}$. As a set, this is all the long knots isotopic to $f$. The primary result of this paper is the computation of the homotopy type of $\mathcal{K}_{f}$ if $f$ is a hyperbolically-spliced knot ie: $f=J \bowtie L$ where $L$ is a hyperbolic KGL. Let $\Sigma_{n}^{+}$be the signed symmetric group. $\Sigma_{n}^{+}$is the semi-direct product $\mathbb{Z}_{2}^{n} \rtimes \Sigma_{n}$ where $\Sigma_{n}$ acts on $\mathbb{Z}_{2}^{n}$ by permutation of factors. Given $L=\left(L_{0}, L_{1}, \cdots, L_{n}\right)$ a hyperbolic KGL, let $B_{L}$ be the group of hyperbolic isometries of the complement of $L$ which extend to orientation-preserving diffeomorphisms of $S^{3}$ such that the extensions to $S^{3}$ preserve $L_{0}$ and its orientation. $B_{L}$ is known to be cyclic by the proof of the Smith Conjecture [31]. There is a natural representation $B_{L} \rightarrow \Sigma_{n}^{+}$given by the composite $B_{L} \rightarrow \operatorname{Diff}\left(S^{3}, L\right) \rightarrow \pi_{0} \operatorname{Diff}\left(\cup_{i=1}^{n} L_{i}\right) \equiv \Sigma_{n}^{+}$. There is an action of $\Sigma_{n}$ on $\mathcal{K}^{n}$ given by permutation of the factors. There is an action of $\mathbb{Z}_{2}$ on $\mathcal{K}$ given by knot inversion, meaning, rotation by $\pi$ about a fixed axis perpendicular to the long axis. These two actions extend in a unique way to an action of $\Sigma_{n}^{+}$on $\mathcal{K}^{n}$. Thus via the representation $B_{L} \rightarrow \Sigma_{n}^{+}$there is an induced action of $B_{L}$ on $\mathcal{K}^{n}$. Let $A_{f}$ be the maximal subgroup of $B_{L}$ such that the induced action of $A_{f}$ on $\mathcal{K}^{n}$ preserves $\prod_{i=1}^{n} \mathcal{K}_{J_{i}}$.

Theorem 1.1 (Proved as Theorem 3.8) If $f=J \bowtie L$ where $L$ is an $(n+1)$-component hyperbolic KGL, then

$$
\mathcal{K}_{f} \simeq S^{1} \times\left(S O_{2} \times A_{f} \prod_{i=1}^{n} \mathcal{K}_{J_{i}}\right)
$$

The composite $A_{f} \rightarrow \operatorname{Diff}\left(S^{3}, L_{0}\right) \rightarrow \operatorname{Diff}\left(L_{0}\right)$ is faithful, giving an embedding $A_{f} \rightarrow S O_{2}$, which is the action of $A_{f}$ on $\mathrm{SO}_{2}$.

This result gives a recursive description of all of the homotopy-type of all of the components of $\mathcal{K}$ since we have the prior results:

(1) [15] If $f$ is the unknot, then $\mathcal{K}_{f}$ is contractible.

(2) [17] If $f$ is a torus knot, then $\mathcal{K}_{f} \simeq S^{1}$.

(3) [17] If $f$ is a hyperbolic knot, then $\mathcal{K}_{f} \simeq S^{1} \times S^{1}$.

(4) [17] If a knot $f$ is a cabling of a knot $g$ then $\mathcal{K}_{f} \simeq S^{1} \times \mathcal{K}_{g}$.

(5) [3] If the knot $f$ is a connected sum of $n \geq 2$ prime knots $f_{1}, f_{2}, \cdots, f_{n}$ then $\mathcal{K}_{f} \simeq$ $\mathcal{C}_{2}(n) \times_{\Sigma_{f}} \prod_{i=1}^{n} \mathcal{K}_{f_{i}} . \mathcal{C}_{2}(n)=\left\{\left(x_{1}, \cdots, x_{n}\right) \in\left(\mathbb{R}^{2}\right)^{n}: x_{i} \neq x_{j} \forall i \neq j\right\} . \quad \Sigma_{f} \subset \Sigma_{n}$ is a Young subgroup of $\Sigma_{n}$, acting on $\mathcal{C}_{2}(n)$ by permutation of the coordinates, and similarly by permuting the factors of the product $\prod_{i=1}^{n} \mathcal{K}_{f_{i}} \cdot \Sigma_{f}$ is the subgroup of $\Sigma_{n}$ preserving the partition of $\{1,2, \cdots, n\}$ defined by the equivalence relation $i \sim j \Longleftrightarrow \mathcal{K}_{f_{i}}=\mathcal{K}_{f_{j}}$.

(6) [4] If a knot has a non-trivial companionship tree, then it is either a cable, in which case (4) applies, a connect-sum, in which case (5) applies, or is hyperbolically spliced, in which case Theorem 1.1 applies. If a knot has a trivial companionship tree, it is either the unknot, in which case (1) applies, or a torus knot in which case (2) applies, or a hyperbolic knot, in which case (3) applies. Moreover, every time one applies one of the above theorems, one reduces the problem of computing the homotopy type of $\mathcal{K}_{f}$ to computing the homotopy type of knotspaces for knots with shorter companionship trees, thus the process terminates. 
Items (2) and (4) are re-proved in Theorem 2.3, (3) is a special case of Theorem 1.1, and (5) is re-proven in Theorem 2.4 .

In Section 3 Theorem 3.8 is proved, giving the homotopy type of $\mathcal{K}_{f}$ when $f=J \bowtie L, L$ a hyperbolic KGL, completing the description of the homotopy type of $\mathcal{K}$, modulo one issue. That being, a recursive description of the homotopy class of the inversion map $I: \mathcal{K}_{f} \rightarrow \mathcal{K}_{f}$ when $f$ is an invertible knot.

In Section 4 a summary is given of how the results of Sections 2 and 3 enable one to compute the homotopy type of $\mathcal{K}$ as an $\mathrm{SO}_{2}$-space, where the $\mathrm{SO}_{2}$ is the subspace of $\mathrm{SO}_{3}$ given by rotations that preserve the 'long axis.' In Theorem 4.4 a recursive computation of the homotopy class of the inversion map is given. These results in turn describe the homotopy type of the space of embeddings of $S^{1}$ in $S^{3}$.

In Section 5 various algorithms and computational methods are mentioned that allow one to apply the theorems of this paper and compute the homotopy type of components of $\mathcal{K}$. Several example computations using SnapPea are given.

Section 6 is devoted to 'the Gramain element.' This is a non-trivial element of $\pi_{1} \mathcal{K}_{f}$ provided $f$ is non-trivial. It was first studied by Gramain [13], and is given by $2 \pi$ rotation around the long axis. The Gramain element survives to give a non-trivial element in $H_{1} \mathcal{K}_{f}$ which is called the Gramain cycle. Thus, the only component of $\mathcal{K}$ with trivial homology is the unknot component.

Section 7 considers several natural questions that the results of this paper raise, such as: what is the relationship to the Vassiliev spectral sequence for $H_{*} \mathcal{K}$, the Goodwillie calculus, the homotopy type of the group-completion of $\mathcal{K}$, and a symmetry realization problem for hyperbolic KGLs related to the problem of giving a 'closed form' description of $H_{*}(\mathcal{K} ; \mathbb{Z})$.

The study of embeddings and to a lesser extent, spaces of embeddings, was initiated by Whitney [50], whose Weak Embedding Theorem could perhaps be viewed as one of the first theorems in the subject. Of course, classical knot theory is an example of the theory of embedding spaces and it predates Whitney, but the focus of the subject historically was not on the global nature of embedding spaces, but more on the 'discrete' isotopy classes of knots.

Perhaps the first 'strong' results on the homotopy type of $\mathcal{K}$ were due to Gramain [13], who used the Burde-Zieschang characterization of the centres of knot groups to show that the map $\pi_{1} S O_{2} \rightarrow \pi_{1} \mathcal{K}_{f}$ given by rotation of a non-trivial long knot about the 'long axis' is injective. Gramain also constructed injections $P_{n} \rightarrow \pi_{1} \mathcal{K}_{f}$ for certain non-prime $f \in \mathcal{K}$ where $P_{n}$ is the pure braid group.

By the work of Hatcher [14] and Ivanov [22, 23] it was known that the Smale Conjecture is equivalent to proving that the unknot component of $\mathcal{K}$ is contractible, which Hatcher later went on to prove [15]. Less well known is one of the consequences: that all components of $\mathcal{K}$ are $K(\pi, 1)$ 's [17]. More recently, Hatcher and McCullough proved [19] that each component of $\mathcal{K}$ has the homotopy type of a finite-dimensional CW-complex. In a sequence of preprints $[17,18]$ Hatcher has also shown, assuming the Linearization Conjecture (that every finite group acting on $S^{3}$ is conjugate to a linear action), how to compute the homotopy type of $\mathcal{K}_{f}$ provided the companionship tree $\mathbb{G}_{f}$ has no branching. Moreover, Hatcher went on to describe the homotopy type of the components of hyperbolic knots and torus knots in $\operatorname{Emb}\left(S^{1}, S^{3}\right) / \operatorname{Diff}\left(S^{1}\right)$.

For the author, it was Victor Tourtchine's conjecture that knot spaces admit actions of the operad of little 2 -cubes that motivated recent work in the subject. After showing that $\mathcal{K}$ admits 
a little 2-cubes action [3] it was only natural to ask if the action was 'free' in the sense of May [29]. The affirmative answer to that question [3] along with some work on JSJ-decompositions [4] led to the realization that the homotopy type of $\mathcal{K}$ is quite explicitly 'computable,' which is the point of this paper.

Tourtchine [43] was working on the homotopy type of spaces of knots from the point of view of singularity theory, as developed by Vassiliev [45]. He described a bracket on the $E^{2}$-page of the Vassiliev spectral sequence for $H_{*} \mathcal{K}$ which conjecturally came from an action of the operad of little 2 -cubes on $\mathcal{K}$. Vassiliev's theory looks at $\mathcal{K}$ as a subspace of a contractible space of maps, $\mathcal{K}$ being seen as the complement of a 'discriminant' space of singular maps, this gives rise to a spectral sequence for the homology of $\mathcal{K}$ via a filtration of the 'discriminant' by a natural notion of 'complexity' of the singularity. Vassiliev's spectral sequence has been, in effect, re-derived by Sinha [39] who studies spaces of knots using the Goodwillie Calculus of Embeddings [12]. Both the approaches of singularity theory and Goodwillie Calculus have technical problems in dimension 3 as the relevant spectral sequences have been shown to not converge to $H_{*} \mathcal{K}$ $[45,46]$. Indeed, most of the literature related to Vassiliev's work focuses on the elements of $H^{0} \mathcal{K}$ produced by the cohomological spectral sequence, the so-called 'Vassiliev invariants' of knots. What relation the results in this paper have to these spectral-sequence approaches to $\mathcal{K}$ is an open problem.

The Goodwillie Calculus methods work very well in co-dimension 3 or larger, as they allow explicit computation of various homotopy groups of knotspaces [38]. Recently Lambrechts, Tourtchine and Volic [27] have reduced the problem of computing the rational homology of such knot spaces to computing the homology of a rather explicit DGA. They do this by showing the rational collapse of the Vassiliev spectral sequence. There are several new interesting developments in the area, such as K. Sakai's homological relationship between $\Omega C_{k}\left(\mathbb{R}^{n}\right)$ and the space of long knots in $\mathbb{R}^{n+1}$ for $n \geq 3$ via 'capping.' In a rather direct application of Goodwillie's dissertation, it is shown that the first non-trivial homotopy groups of the space of 'long embeddings' of $\mathbb{R}^{j}$ in $\mathbb{R}^{n}$ are related via a generalized Litherland Spinning construction provided $2 n-3 j-3 \geq 0[5]$. Among other things this result provides a geometric isomorphism between $\pi_{2}$ (the space of long knots in $\left.\mathbb{R}^{4}\right)$ and $\pi_{0} \operatorname{Emb}\left(S^{3}, S^{6}\right) \simeq \mathbb{Z}$.

I thank Allen Hatcher for his input on this project. I thank Fred Cohen. This paper probably never would have been finished if it wasn't for his frequent encouragement. I'd like to thank Cameron Gordon for pointing out an error in an early attempt at a proof of Theorem 3.8 and for bringing the Hartley paper to my attention [20]. I owe thanks to Jeff Weeks, indirectly, for his wonderful software, SnapPea. I thank Morwen Thistlethwaite for showing me how to convince SnapPea to compute the symmetry groups $B_{L}$ in Theorem 3.8. I thank Yoshiaki Uchida for his input. I also thank the University of Oregon's topology/geometry seminar, particularly Dev

Sinha, Hal Sadofsky, and Gregor Masbaum for listening to early versions of these results, and the resulting helpful conversations. I would also like to thank the Max Planck Institute for Mathematics and Institut des Hautes Études Scientifiques for support during the preparation of this manuscript.

\section{The recursive setup for the homotopy-type of $\mathcal{K}$}

This section reviews the techniques for describing the homotopy-type of $\mathcal{K}$, quoting and reproving various theorems about embedding spaces. 
Theorem 2.1 Given a long knot $f \in \mathcal{K}$, consider the one-point compactification of $f$ to be an embedding of $S^{1}$ in $S^{3}$ and let $C$ be the complement of an open tubular neighbourhood of this embedding. The restriction map is a Serre fibration

$$
\operatorname{Diff}\left(D^{3} \text { fix } \partial D^{3}\right) \rightarrow \mathcal{K}_{f}
$$

where $\operatorname{Diff}\left(D^{3}\right.$ fix $\left.\partial D^{3}\right)$ is the group of diffeomorphisms of $D^{3}$ which fix the boundary disc pointwise.

(1) [17] The fibre of the map $\operatorname{Diff}\left(D^{3}\right.$ fix $\left.\partial D^{3}\right) \rightarrow \mathcal{K}_{f}$ has the homotopy type of $\operatorname{Diff}(C$ fix $\partial C)$, the group of diffeomorphisms of $C$ that fix the boundary pointwise.

(2) $\quad$ [15] $\operatorname{Diff}\left(D^{3}\right.$ fix $\left.\partial D^{3}\right)$ is contractible, thus $\mathcal{K}_{f} \simeq B \operatorname{Diff}(C$ fix $\partial C)$.

(3) [14] $\operatorname{Diff}(C$ fix $\partial C)$ has the homotopy-type of a discrete group. Equivalently, $\mathcal{K}_{f}$ is a $K(\pi, 1)$.

Given any manifold $M$ and a submanifold $N$, that the restriction map $\operatorname{Diff}(M) \rightarrow \operatorname{Emb}(N, M)$ is a Serre fibration is a classical theorem. The homotopy lifting property for this map is proved by observing that in the classical proof of the Isotopy Extension Theorem for an isotopy of $N$ in $M$, the solution depends smoothly on the initial conditions (the embedding of $N$ in $M$ ), as the isotopy extension is constructed by solving an ordinary differential equation. A specific reference is Cerf's paper [9] $\S 2.2 .2$, but perhaps it is preferable for the reader to adapt Hirsch's textbook proof of isotopy extension [21] §8.1.3. Point (1) in Proposition 2.1 follows from the homotopy classification of spaces of tubular neighbourhoods, see [9] or adapt the isotopy-classification of tubular neighbourhoods from [21]. Point (2) is a difficult theorem of Hatcher's, historically called 'the Smale Conjecture' [15], and point (3) is a consequence of (2) when combined with the theorems, proved independently by Hatcher and Ivanov, on the homotopy-type of spaces of incompressible surfaces in 3-manifolds [14, 22, 23].

Lemma 2.2 Let $f$ be a compound knot, ie: $f=\left(f_{1}, \cdots, f_{n}\right) \bowtie L$ with $L$ a KGL. There is an exact sequence of groups

$$
0 \rightarrow \prod_{i=1}^{n} \pi_{0} \operatorname{Diff}\left(C_{f_{i}} \text { fix } \partial C_{f_{i}}\right) \rightarrow \pi_{0} \operatorname{Diff}(C \text { fix } \partial C) \rightarrow \pi_{0} \operatorname{Diff}(V \text { fix } \partial C)
$$

where $\overline{C \backslash V}=\sqcup_{i=1}^{n} C_{f_{i}}$, ie: $C_{f_{i}}$ is diffeomorphic to the complement of a non-trivial knot $f_{i} . V$ is canonically diffeomorphic to the complement of the link $L$, denoted $C_{L}$.

Proof The key technique is to observe that $\operatorname{Diff}(C$ fix $\partial C)$ deformation-retracts to the subgroup which preserves $V$, using the incompressible surface results in [14] or [22, 23]. Restriction to $V$ gives a fibration whose homotopy long exact sequence is the exact sequence in the lemma.

Since there is a canonical diffeomorphism $V \rightarrow C_{L}$ (called the untwisted re-embedding in [4]), let $T_{0} \subset \partial C_{L}$ correspond to $\partial C \subset V$ under this diffeomorphism. Observe that image of the map $\pi_{0} \operatorname{Diff}(C$ fix $\partial C) \rightarrow \pi_{0} \operatorname{Diff}(V$ fix $\partial C) \equiv \pi_{0} \operatorname{Diff}\left(C_{L}\right.$ fix $\left.T_{0}\right)$ consists precisely of the elements of $\operatorname{Diff}\left(C_{L}\right.$ fix $\left.T_{0}\right)$ which extend to diffeomorphisms of the pair $\left(S^{3}, L\right)$ such that (a) if $L_{i}$ is sent to $L_{j}$ preserving the orientations, then $f_{i}$ is isotopic to $f_{j}$, and (b) if $L_{i}$ is sent to $-L_{j}$, then $f_{i}$ is isotopic to the inverse of $f_{j}$. The conventions of Lemma 2.2 will be used throughout the remainder of the paper. 
Theorem 2.3 If $f \in \mathcal{K}$ is a cable of $g \in \mathcal{K}$ then $\mathcal{K}_{f} \simeq S^{1} \times \mathcal{K}_{g}$.

Proof There are two cases to consider:

(1) The JSJ-tree has only one vertex. This is the case where $f$ is a non-trivial torus knot, $g$ is the unknot, and $V=C$.

(2) The JSJ-tree has two or more vertices. This is the case that $f$ is a cable of a non-trivial knot $g$ and $V$ is diffeomorphic to the complement of a 2-component Seifert-fibred link complement in $S^{3}$.

In case $(1), \pi_{0} \operatorname{Diff}(C$ fix $\partial C) \simeq \mathbb{Z}$. This is because $C$ is a Seifert-fibred manifold, fibred over a disc with two singular fibres. The singular fibres have Seifert-fibre data $\frac{\alpha_{1}}{\beta_{1}}$ and $\frac{\alpha_{2}}{\beta_{2}}$ where $\alpha_{1} \beta_{2}+\alpha_{2} \beta_{1}= \pm 1$, in the 'unnormalized' notation of Hatcher $C \simeq M\left(0,1 ; \frac{\alpha_{1}}{\beta_{1}}, \frac{\alpha_{2}}{\beta_{2}}\right)[16]$. Any diffeomorphism of $C$ can be isotoped to one which preserves the Seifert-fibring [14], thus $\pi_{0} \operatorname{Diff}(C)$ is generated by a meridional Dehn twist about a boundary-parallel torus. Such Dehn twists are non-trivial since all non-trivial powers of the meridian are non-central in $\pi_{1} C$ [8], thus $\mathcal{K}_{f} \simeq B \operatorname{Diff}(C) \simeq S^{1}$ and $\pi_{1} \mathcal{K}_{f}$ is generated by full rotation about the long axis of the knot.

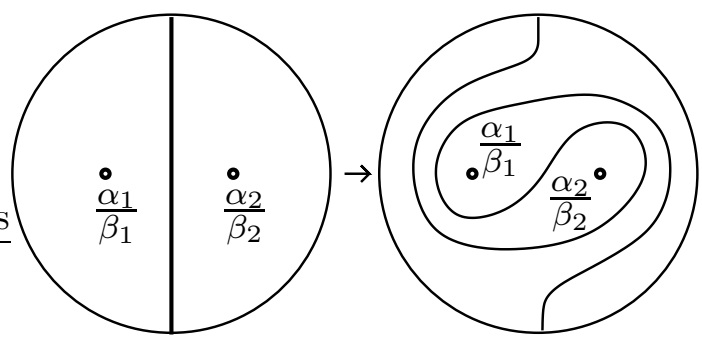

The generator of $\pi_{0} \operatorname{Diff}(C) \simeq \pi_{1} \mathcal{K}_{f}$

In case (2) $V \simeq M\left(0,2 ; \frac{\alpha_{1}}{\beta_{1}}\right)$ is Seifert fibred over a once-punctured disc with one singular fibre. The map $\pi_{0} \operatorname{Diff}(C$ fix $\partial C) \rightarrow \pi_{0} \operatorname{Diff}(V$ fix $\partial C)$ is onto an infinite-cyclic group generated by a meridional Dehn twist about a torus parallel to $\partial C$ via an argument analogous to case (1). This allows the direct construction of an isomorphism $\pi_{0} \operatorname{Diff}\left(C_{g}\right.$ fix $\left.\partial C_{g}\right) \times \mathbb{Z} \rightarrow \pi_{0} \operatorname{Diff}(C$ fix $\partial C)$ by taking the union of a diffeomorphism on $C_{g}$ with a Dehn twist on $V$.

Theorem 2.4 If $f$ is a connect sum of prime knots $f_{1}, \cdots, f_{n}$ then

$$
\mathcal{K}_{f} \simeq \mathcal{C}_{2}(n) \times_{\Sigma_{f}} \prod_{i=1}^{n} \mathcal{K}_{f_{i}}
$$

where $\Sigma_{f} \subset \Sigma_{n}$ is the Young subgroup corresponding to the equivalence relation $i \sim j$ iff $f_{i}$ and $f_{j}$ are isotopic as long knots.

Proof The proof follows the outline of Theorem 2.3, but in this case $V \simeq C_{L}$ where $L$ is the $(n+1)$-component keychain link. The mapping class group of diffeomorphisms of the pair $\left(S^{3}, L\right)$ which fix a tubular neighbourhood of $L_{0}$ pointwise is isomorphic to the braid group, since $C_{L}$ is diffeomorphic to a product of a circle with an $n$-punctured disc [14]. Thus the image of the map $\pi_{0} \operatorname{Diff}(C$ fix $\partial C) \rightarrow \pi_{0} \operatorname{Diff}\left(C_{L}\right.$ fix $\left.T_{0}\right)$ corresponds to the preimage of $\Sigma_{f}$ under the canonical epi-morphism $B_{n} \rightarrow \Sigma_{n}$ where $B_{n}$ is the braid group on $n$-strands. Constructing a splitting over this group amounts to choosing a canonical identification between $C_{f_{i}}$ and $C_{f_{j}}$ for all $f_{i}$ isotopic to $f_{j}$ and extending via this identification.

In [3] it took quite a bit more work to prove Theorem 2.4 since it involved showing that an action of the operad of 2 -cubes gave the splitting. 


\section{The homotopy type of a hyperbolically spliced knot}

Let $V$ be an orientable, complete cusped hyperbolic manifold of finite volume. Let $\bar{V}$ denote the manifold compactification of $V$, ie: if $V$ has $n$ cusps, then $\bar{V}$ has $n$ torus boundary components.

Definition 3.1 Let $\operatorname{Isom}(V)$ be the group of hyperbolic isometries of $V$. Any isometry of $V$ extends to a diffeomorphism of $\bar{V}$ since in a horoball neighbourhood of a cusp the isometry is a Euclidean motion. This gives a map $\operatorname{Isom}(V) \rightarrow \operatorname{Diff}(\bar{V})$. Let $\operatorname{HomEq}(V)$ be the space of homotopy equivalences of $V$. There is a natural map $\operatorname{Diff}(\bar{V}) \rightarrow \operatorname{HomEq}(V)$ given by restriction to $V$.

Proposition 3.2 The maps $\operatorname{Isom}(V) \rightarrow \operatorname{Diff}(\bar{V})$ and $\operatorname{Diff}(\bar{V}) \rightarrow \operatorname{HomEq}(V)$ are homotopy equivalences.

Proof Mostow rigidity [32] tells us that the map $\operatorname{Isom}(V) \rightarrow \operatorname{HomEq}(V)$ is a homotopy equivalence. By the work of Hatcher and Ivanov [14, 22, 23], Diff $(\bar{V})$ is a $\mathcal{K}(\pi, 0)$. Thus we need only show that $\pi_{0} \operatorname{Diff}(\bar{V}) \rightarrow \pi_{0} \operatorname{HomEq}(V)$ is injective. Waldhausen [47] (Lemma 1.4.2) proves homotopic diffeomorphisms of Haken manifolds are isotopic provided the homotopy preserves the boundary of the manifolds. In his Theorem 7.1 [47] he proves this boundary condition can always be satisfied for boundary-irreducible manifolds. We can disregard the special case of $I$-bundles as they are not cusped hyperbolic manifolds of finite volume.

Let $L=\left(L_{0}, L_{1}, \cdots, L_{n}\right)$ be an $(n+1)$-component hyperbolic KGL, and let $C_{L}$ be its complement. Let $T_{0}$ be the boundary of $C_{L}$ corresponding to the $L_{0}$-cusp. Note that an isometry of $C_{L}$ extends to a diffeomorphism of $S^{3}$ if and only if it preserves the longitudes and meridians of $L$. Moreover, if $G$ is any subgroup of $\operatorname{Isom}\left(C_{L}\right)$ consisting of elements which extend to diffeomorphisms of $S^{3}$, then the action of $G$ on $C_{L}$ extends to an action of $G$ on the pair $\left(S^{3}, L\right)$. This action can be constructed 'by hand' using the fact that $G$ acts by Euclidean motions on the boundary tori of $C_{L}$.

Definition 3.3 Let $B_{L}$ be the subgroup of $\operatorname{Isom}\left(C_{L}\right)$ that both extend to diffeomorphisms of $S^{3}$ and act on the $L_{0}$-cusp by translations, where we identify $\partial C_{L}$ with the boundary of some tubular neighbourhood of $L$ in $S^{3}$. Let $\operatorname{Trans}\left(T_{0}\right)$ be the group of Euclidean translations of the $L_{0}$-cusp $T_{0}$. Basic Riemmanian geometry tells us that $B_{L} \rightarrow \operatorname{Trans}\left(T_{0}\right)$ is an embedding. As a group $\operatorname{Trans}\left(T_{0}\right)$ is isomorphic to $S O_{2}^{2}$. Let $\widetilde{\operatorname{Trans}}\left(T_{0}\right) \rightarrow \operatorname{Trans}\left(T_{0}\right)$ be the universal covering space, and let $\widetilde{B_{L}}$ be the lift of $B_{L}$ to the universal cover. Let $\operatorname{Diff}_{T_{0}}\left(C_{L}\right)$ be the group of diffeomorphisms of $C_{L}$ which both extend to diffeomorphisms of $S^{3}$ and restrict to diffeomorphisms of $T_{0}$ which are isotopic to the identity. Let $\operatorname{Diff}\left(C_{L}\right.$ fix $\left.T_{0}\right)$ be the subgroup of $\operatorname{Diff}_{T_{0}}\left(C_{L}\right)$ which fixes $T_{0}$ pointwise.

Proposition $3.4 \pi_{0} \operatorname{Diff}\left(C_{L}\right.$ fix $\left.T_{0}\right)$ is free abelian on two generators. Moreover the map $\operatorname{Diff}\left(C_{L}\right.$ fix $\left.T_{0}\right) \rightarrow \operatorname{Diff}_{T_{0}}\left(C_{L}\right)$ is equivalent to the projection $\widetilde{B_{L}} \rightarrow B_{L}$.

Proposition 3.4 is proven as Proposition 3.2 in [19], so the proof is omitted here. But it is enlightening to understand the equivalence $\xi: \widetilde{B_{L}} \rightarrow \operatorname{Diff}\left(C_{L}\right.$ fix $\left.T_{0}\right)$. If $\tilde{f} \in \widetilde{B_{L}}$, let $f \in$ $\operatorname{Isom}\left(C_{L}\right)$ be the corresponding isometry, then $\xi(\tilde{f}) \in \operatorname{Diff}\left(C_{L}\right.$ fix $\left.T_{0}\right)$ is defined to be equal to 
$f$ outside a fixed collar neighbourhood of $T_{0}$. Extend $\xi(\tilde{f})$ to be a diffeomorphism of $C_{L}$ by the straight line from $0 \in \widetilde{\operatorname{Trans}}\left(T_{0}\right)$ to $\tilde{f} \in \widetilde{\operatorname{Trans}}\left(T_{0}\right)$. Such a path gives a path of translations of $T_{0}$ thus a fibre-preserving diffeomorphism of the collar neighbourhood of $T_{0}$ starting at the identity and ending at a map which agrees with the isometry $f$.

For $L=\left(L_{0}, L_{1}, \cdots, L_{n}\right)$ a KGL, let $\operatorname{Diff}\left(S^{3}, L, L_{0}\right)$ be the group of diffeomorphisms of $S^{3}$ which preserve $L$, and which also preserve $L_{0}$ together with its orientation.

Proposition 3.5 (No Bad Monodromy) Let $L$ be a hyperbolic KGL, then the composite $B_{L} \rightarrow \operatorname{Diff}\left(S^{3}, L, L_{0}\right) \rightarrow \operatorname{Diff}\left(L_{0}\right)$ has a trivial kernel. Equivalently, $B_{L} \rightarrow \operatorname{Diff}\left(L_{0}\right)$ is an embedding.

Proof Let $H$ be the kernel of the composite $B_{L} \rightarrow \operatorname{Diff}\left(S^{3}, L, L_{0}\right) \rightarrow \operatorname{Diff}\left(L_{0}\right)$. For $n=0$ this is true since if $H$ was non-trivial, $L=\left(L_{0}\right)$ is a hyperbolic knot that's a fixed point set of a finite group action on $S^{3}$ which would contradict the Smith conjecture [31].

For $n \geq 1$ if $H$ was non-trivial, $L_{0}$ would be the unknot and $S^{3} / H$ can be identified with $S^{3}$ by the resolution of the Smith conjecture [31]. The quotient $\left(S^{3}, L\right) / H$ is a manifold pair $\left(S^{3} / H, L / H\right)$ where $S^{3} / H \simeq S^{3}$ and $L / H \subset S^{3} / H$ is some link. We identify $S^{3} / H$ with $S^{3}$ and $L / H$ with $L^{\prime}=\left(L_{0}^{\prime}, L_{1}^{\prime}, \cdots, L_{p}^{\prime}\right) \subset S^{3}$. Notice that $S^{3} \backslash L^{\prime}$ is hyperbolic.

By definition, there is an epimorphism $\pi_{1}\left(S^{3} \backslash L_{0}^{\prime}\right) \rightarrow H$. Define a homomorphism $\pi_{1}\left(S^{3} \backslash L^{\prime}\right) \rightarrow$ $\mathbb{Z}_{2}$ to be the sum of the mod-2 linking numbers of a path in $S^{3} \backslash L^{\prime}$ with the link components $L^{\prime} \backslash L_{0}^{\prime}$. Thus we have an epimorphism $\pi_{1}\left(S^{3} \backslash L^{\prime}\right) \rightarrow \mathbb{Z}_{2} \oplus H$ given by the direct-sum of the above two homomorphisms.

If we take the abelian branched cover $\pi: X \rightarrow S^{3}$ corresponding to the above homomorphism $\pi_{1}\left(S^{3} \backslash L^{\prime}\right) \rightarrow \mathbb{Z}_{2} \oplus H$, the branch-point set is precisely $L^{\prime}$, and since the homomorphism $\pi_{1}\left(S^{3} \backslash L^{\prime}\right) \rightarrow \mathbb{Z}_{2} \oplus H$ is a direct sum, the covering map $\left(X, \pi^{-1} L^{\prime}\right) \rightarrow\left(S^{3}, L^{\prime}\right)$ factors as a composite $\left(X, \pi^{-1} L^{\prime}\right) \rightarrow\left(S^{3}, L\right) \rightarrow\left(S^{3}, L^{\prime}\right)$ where the map $\left(X, \pi^{-1} L^{\prime}\right) \rightarrow\left(S^{3}, L\right)$ is an abelian branched cover with branch-point set $L \backslash L_{0}$.

Since $L \backslash L_{0}$ is an $n$-component unlink, this forces $X$ to be a connect-sum of copies of $S^{1} \times S^{2}$. Precisely,

$$
X \simeq \#_{n-1} S^{1} \times S^{2}
$$

Sakuma's Theorem B [36] tells us that $L^{\prime}$ is either a trivial knot or a connect-sum of Hopf links, or is split. Since $L^{\prime}$ is hyperbolic it can be none of these, contradicting our assumption that $H$ was non-trivial.

Definition 3.6 Let $I: \mathcal{K} \rightarrow \mathcal{K}$ be the involution of $\mathcal{K}$ induced by rotation by $\pi$ around an axis perpendicular to the long axis. A knot $f \in \mathcal{K}$ is said to be invertible if $I\left(\mathcal{K}_{f}\right)=\mathcal{K}_{f}$. The knot $f$ is strongly invertible if $I: \mathcal{K}_{f} \rightarrow \mathcal{K}_{f}$ has a fixed point.

Not all knots are invertible [44], and not all invertible knots are strongly invertible [20, 1]. $\mathbb{Z}_{2}=\{ \pm 1\}$ acts on $\mathcal{K}$ by inversion (rotation by $\pi$ about any axis perpendicular to the long axis). $\Sigma_{n}$ acts on $\mathcal{K}^{n}$ by permutation of the factors. These two actions induce an action of $\Sigma_{n}^{+}=\{ \pm 1\}^{n} \rtimes \Sigma_{n}$ on $\mathcal{K}^{n}$. Let $f=J \bowtie L$ where $L$ is an $(n+1)$-component hyperbolic KGL, then $B_{L}$ has a natural representation into $\Sigma_{n}^{+}$given by the composite $B_{L} \rightarrow \operatorname{Diff}\left(S^{3}, L, L_{0}\right) \rightarrow$ $\pi_{0} \operatorname{Diff}\left(L_{1} \cup \cdots \cup L_{n}\right) \equiv \Sigma_{n}^{+}$. 
Definition 3.7 $A_{f}$ is the subgroup of $B_{L}$ that preserves $\prod_{i=1}^{n} \mathcal{K}_{J_{i}}$ under the $B_{L}$ action on $\mathcal{K}^{n}$.

Theorem 3.8 Let $f=J \bowtie L$, where $L$ is a hyperbolic KGL, then there is a homotopyequivalence:

$$
\mathcal{K}_{f} \simeq\left(\prod_{i=1}^{n} \mathcal{K}_{J_{i}}\right) \times_{A_{f}} \operatorname{Trans}\left(T_{0}^{\prime}\right)
$$

and there is a further splitting

$$
\mathcal{K}_{f} \simeq S^{1} \times\left(\left(\prod_{i=1}^{n} \mathcal{K}_{J_{i}}\right) \times_{A_{f}} L_{0}\right)
$$

Proof Let $\tilde{A}_{f} \rightarrow A_{f}$ be the pull-back of $\tilde{B}_{L} \rightarrow B_{L}$ via the inclusion $A_{f} \subset B_{L}$. By Proposition 3.2 and the observations in Lemma 2.2 the map $\pi_{0} \operatorname{Diff}(C$ fix $\partial C) \rightarrow \pi_{0} \operatorname{Diff}\left(C_{L}\right.$ fix $\left.T_{0}\right) \equiv \tilde{B}_{L}$ is onto $\tilde{A}_{f}$. By Proposition 3.5, $\tilde{A}_{f}$ is free abelian, generated by two elements, with one being a meridional Dehn Twist. This allows us to construct a splitting of the map $\pi_{0} \operatorname{Diff}(C$ fix $\partial C) \rightarrow$ $\tilde{A}_{f}$ in two steps: 1) choose an element in the preimage of the generator which is not the meridional Dehn Twist. 2) The meridional Dehn Twist in $\pi_{0} \operatorname{Diff}\left(C_{L}\right.$ fix $\left.T_{0}\right)$ has a meridional Dehn twist in $\pi_{0} \operatorname{Diff}(C$ fix $\partial C)$ as a preimage, which is central. The (1) and (2) prescribe a section of the whole group $\tilde{A}_{f}$.

This forces $\pi_{0} \operatorname{Diff}\left(C\right.$ fix $\partial C$ ) to be the semi-direct product of $\tilde{A}_{f}$ and $\prod_{i=1}^{n} \pi_{0} \operatorname{Diff}\left(C_{J_{i}}\right.$ fix $\left.\partial C_{J_{i}}\right)$. The action of $\tilde{A}_{f}$ on $\prod_{i=1}^{n} \pi_{0} \operatorname{Diff}\left(C_{J_{i}}\right.$ fix $\left.\partial C_{J_{i}}\right)$ (by conjugation) sends $\operatorname{Diff}\left(C_{J_{i}}\right.$ fix $\left.\partial C_{J_{i}}\right)$ to $\operatorname{Diff}\left(C_{J_{k}}\right.$ fix $\left.\partial C_{J_{k}}\right)$ if the corresponding permutation $\tilde{A}_{f} \rightarrow A_{f} \rightarrow \Sigma_{n}^{+}$sends $i$ to $k$. If the permutation sends $i$ to $k$ preserving the sign, this means the $J_{i}$ and $J_{k}$ are isotopic as long knots and $\operatorname{Diff}\left(C_{J_{i}}\right.$ fix $\left.\partial C_{J_{i}}\right) \rightarrow \operatorname{Diff}\left(C_{J_{k}}\right.$ fix $\left.\partial C_{J_{k}}\right)$ is conjugation by a diffeomorphism $C_{J_{i}} \rightarrow C_{J_{k}}$ that can be realized by an isotopy from the long knot $J_{i}$ to $J_{k}$. If on the other hand the sign is not preserved, then the map $\operatorname{Diff}\left(C_{J_{i}}\right.$ fix $\left.\partial C_{J_{i}}\right) \rightarrow \operatorname{Diff}\left(C_{J_{k}}\right.$ fix $\left.\partial C_{J_{k}}\right)$ is conjugation by a map $C_{J_{i}} \rightarrow C_{J_{k}}$ which can be realized by an isotopy in $\mathbb{R}^{3}$ that reverses the long axis of the knot. Apply the classifying space functor $B$ to these results and the theorem follows.

It's interesting to compare this result to a theorem that appears in Hatcher's papers $[17,18]$ on the homotopy type of the component of a hyperbolic knot $f$ in $\operatorname{Emb}\left(S^{1}, S^{3}\right) / \operatorname{Diff}\left(S^{1}\right)$. Provided the Linearization Conjecture is true, one can put $f$ into a 'maximally-symmetric position,' which means that $f$ has a position so that the restriction map $\operatorname{Isom}_{S^{3}}\left(S^{3}, f\right) \rightarrow \operatorname{Isom}_{\mathbb{H}^{3}}\left(S^{3} \backslash f\right)$ is an isomorphism. $\operatorname{Isom}_{S^{3}}\left(S^{3}, f\right)$ is the group of isometries of $S^{3}$ that preserve the knot, and $\operatorname{Isom}_{\mathbb{H}^{3}}\left(S^{3} \backslash f\right)$ is the group of hyperbolic isometries of $S^{3} \backslash f$. Let $\operatorname{Isom}_{S^{3}}^{+}\left(S^{3}, f\right)$ be the subgroup of $\operatorname{Isom}_{S^{3}}\left(S^{3}, f\right)$ which preserves the orientation of $S^{3}$, thus $\operatorname{Isom}_{S^{3}}^{+}\left(S^{3}, f\right) \subset S O_{4}$. Hatcher's theorem is that the component of $f \in \operatorname{Emb}\left(S^{1}, S^{3}\right) / \operatorname{Diff}\left(S^{1}\right)$ has the homotopy type of $S O_{4} / \operatorname{Isom}_{\mathbb{H}^{3}}^{+}\left(S^{3} \backslash f\right)$, where the representation $\operatorname{Isom}_{\mathbb{H}^{3}}^{+}\left(S^{3} \backslash f\right)=\operatorname{Isom}_{S^{3}}^{+}\left(S^{3}, f\right) \rightarrow S O_{4}$ is given by the inclusion map. So not only does the homotopy type of the components of $\operatorname{Emb}\left(S^{1}, S^{3}\right) / \operatorname{Diff}\left(S^{1}\right)$ depend on the isometry groups $\operatorname{Isom}_{\mathbb{H}^{3}}^{+}\left(S^{3} \backslash f\right)$, but also on the representation $\operatorname{Isom}_{S^{3}}^{+}\left(S^{3}, f\right) \rightarrow S O_{4}$.

Observe that the representation $B_{L} \rightarrow \Sigma_{n}^{+}$is generally not faithful. We will give some examples, with $L=\left(L_{0}, L_{1}, \cdots, L_{n}\right)$ a hyperbolic KGL.

- For $n=0$ the figure-8 knot gives $B_{L}=\mathbb{Z}_{2}$ yet $B_{L} \rightarrow \Sigma_{0}^{+}$is trivial. 
- For $n=1$ the link called $6_{3}^{2}$ in Rolfsen's tables satisfies $B_{L}=\mathbb{Z}_{2}$ with $B_{L} \rightarrow \Sigma_{1}^{+}$trivial.

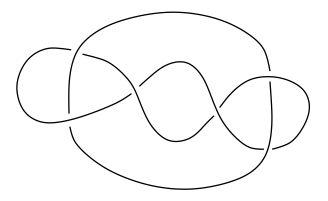

- For $n=2$ in the example below, $B_{L}=\mathbb{Z}_{4}$ and the kernel of $B_{L} \rightarrow \Sigma_{2}^{+}$has order 2

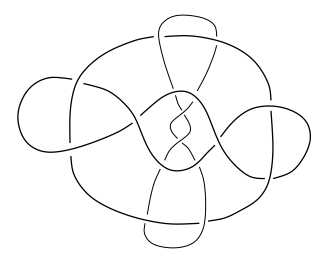

Notice that in order to compute the homotopy type of $\mathcal{K}_{f}$, if $A_{f} \rightarrow \Sigma_{n}^{+}$is not conjugate to a representation $A_{f} \rightarrow \Sigma_{n}$, then we must know some of the spaces $\mathcal{K}_{J_{i}}$ as $O_{2}$-spaces. Minimally, we need to know the homotopy class of the inversion map. In the next section we will inductively study the $\mathrm{O}_{2}$ homotopy type of $\mathcal{K}$, and recursively compute the homotopy class of the inversion map.

\section{Closed knots in $S^{3}$ and the $O_{2}$-action on $\mathcal{K}$}

This section describes the homotopy type of the space of embeddings $\operatorname{Emb}\left(S^{1}, S^{3}\right)$. This problem is reduced to computing the homotopy type of $\mathcal{K}$ as an $\mathrm{O}_{2}$-space, where $\mathrm{O}_{2} \subset \mathrm{SO}_{3}$ is the 'long axis' preserving subgroup.

Proposition $4.1[7] \operatorname{Emb}\left(S^{1}, S^{3}\right) \simeq \mathrm{SO}_{4} \times_{\mathrm{SO}_{2}} \mathcal{K}$. Moreover, there is a homotopy equivalence

$$
\mathrm{SO}_{4} \times \mathrm{SO}_{2} \mathcal{K} \simeq \mathrm{S}^{3} \times\left(\mathrm{SO}_{3} \times \mathrm{SO}_{2} \mathcal{K}\right)
$$

This makes $\mathrm{SO}_{3} \times \mathrm{SO}_{2} \mathcal{K}$ the $\mathcal{K}$-bundle over $\mathrm{S}^{2}$ whose monodromy is induced by the monodromy for the tangent bundle of $S^{2}$.

Consequently, to describe the homotopy of the space of embeddings $\operatorname{Emb}\left(S^{1}, S^{3}\right)$ we need to know the homotopy type of $\mathcal{K}$ as an $\mathrm{SO}_{2}$-space. As we've seen, we can use Theorem 3.8 to compute the homotopy type of the components of $\mathcal{K}$ provided we know the homotopy type of simpler components of $\mathcal{K}$, the $\mathrm{SO}_{2}$-action on those components, and the homotopy class of the inversion involution $I: \mathcal{K} \rightarrow \mathcal{K}$ on those simpler components (provided the knot is invertible). So in this section we 'kill two birds with one stone,' and study $\mathcal{K}$ as an $O_{2}$-space.

Proposition 4.2 ( $\mathrm{O}_{2}$-action on a cabling) If $f$ is a cable of $g$ then

- There is an $\mathrm{SO}_{2}$-equivariant homotopy equivalence

$$
S^{1} \times \mathcal{K}_{g} \rightarrow \mathcal{K}_{f}
$$

Where the $\mathrm{SO}_{2}$ action on $S^{1} \times \mathcal{K}_{g}$ is given by $A \cdot(v, x)=(A \cdot v, x)$. 
- $I\left(\mathcal{K}_{f}\right)=\mathcal{K}_{f}$ if and only if $I\left(\mathcal{K}_{g}\right)=\mathcal{K}_{g}$. If $I\left(\mathcal{K}_{f}\right)=\mathcal{K}_{f}$ then the above homotopy equivalence is $O_{2}$-equivariant where $A \cdot(v, x)=\left(A \cdot v, I^{\epsilon(A)}(x)\right)$. Where $\epsilon: O_{2} \rightarrow\{ \pm 1\}$ is the unique epimorphism.

Proof Consider $f$ to be a splice knot $f=g \bowtie L$ where $L=S^{(p, q)}$ is a 2-component Seifert link [4]. Theorem 2.3 gives us a homotopy-equivalence $S O_{2} \times \mathcal{K}_{g} \rightarrow K_{f}$. We will construct this map explicitly on the space-level. Consider the space of embeddings of $\mathbb{R} \sqcup S^{1}$ in $\mathbb{R}^{3}$ such that the restriction to $\mathbb{R}$ is a long knot. Denote this space by $\operatorname{Emb}\left(\mathbb{R} \sqcup S^{1}, \mathbb{R}^{3}\right)$. Notice that the Theorem 2.1 generalizes immediately to say that the component of $\operatorname{Emb}\left(\mathbb{R} \sqcup S^{1}, \mathbb{R}^{3}\right) / \operatorname{Diff}\left(S^{1}\right)$ that contains the Seifert link $S^{(p, q)}$ has the homotopy-type of $S^{1}$. Moreover, by the same argument as in Theorem 2.3, this component has the $\mathrm{SO}_{2}$-equivariant homotopy-type of $S^{1}$ with the standard $S_{2}$ action. Since $f=g \bowtie S^{(p, q)}$, we can use parametrized splicing [4] to construct an $\mathrm{SO}_{2}$-equivariant map $S^{1} \times \mathcal{K}_{g} \rightarrow \mathcal{K}_{f}$ which is also by design a homotopy-equivalence.

Proposition 4.2 also applies to a torus knot component. In this case, it gives the result that a torus knot component has the homotopy type of $S^{1}$ and the $\mathrm{O}_{2}$-action on $S^{1}$ is precisely the standard action of $\mathrm{O}_{2}$ on $S^{1}$. The next proposition assumes familiarity with the action of the operad of 2 -cubes on $\mathcal{K}[3]$.

\section{Proposition 4.3 ( $\mathrm{O}_{2}$-action on a connected-sum)}

- By [3], if $f=f_{1} \# \cdots \# f_{n}$ where $\left\{f_{i}: i \in\{1,2, \cdots, n\}\right\}$ are the prime summands of $f$, then there is a canonical homotopy equivalence

$$
\mathcal{C}_{2}(n) \times_{\Sigma_{f}} \prod_{i=1}^{n} \mathcal{K}_{f_{i}} \rightarrow \mathcal{K}_{f}
$$

This homotopy equivalence is $\mathrm{SO}_{2}$-equivariant, where the $\mathrm{SO}_{2}$ action on $\mathcal{C}_{2}(n) \times_{\Sigma_{f}}$ $\prod_{i=1}^{n} \mathcal{K}_{f_{i}}$ is given by

$$
A \cdot\left(\left(C_{1}, \cdots, C_{n}\right),\left(g_{1}, \cdots, g_{n}\right)\right)=\left(\left(C_{1}, \cdots, C_{n}\right),\left(A \cdot g_{1}, \cdots, A \cdot g_{n}\right)\right)
$$

- The $\mathrm{O}_{2}$ action on $\mathcal{K}$ restricts to $\mathcal{K}_{f}$ if and only if there is some involution $\iota \in \Sigma_{n}$ so that $\mathcal{K}_{I f_{i}}=\mathcal{K}_{f_{\iota(i)}}$ for all $i \in\{1,2, \cdots, n\}$. In this case the map $\mathcal{C}_{2}(n) \times_{\Sigma_{f}} \prod_{i=1}^{n} \mathcal{K}_{f_{i}} \rightarrow \mathcal{K}_{f}$ is $\mathrm{O}_{2}$-equivariant where the $\mathrm{O}_{2}$ action on $\mathcal{C}_{2}(n) \times{ }_{\Sigma_{f}} \prod_{i=1}^{n} \mathcal{K}_{f_{i}}$ is given by

$$
A \cdot\left(\left(C_{1}, \cdots, C_{n}\right),\left(g_{1}, \cdots, g_{n}\right)\right)=\left(\epsilon(A) \cdot\left(C_{\iota(1)}, \cdots, C_{\iota(n)}\right),\left(A \cdot g_{\iota(1)}, \cdots, A \cdot g_{\iota(n)}\right)\right)
$$

where $\epsilon(A) \in\{ \pm 1\}$ acts on $\mathcal{C}_{2}(n)$ by mirror reflection about the $y$-axis and $\epsilon: O_{2} \rightarrow\{ \pm 1\}$ is the unique epimorphism.

Proof The homotopy equivalence is the little 2 -cubes action $\kappa$ [3]. To check equivariance, we use the 'trivially framed' space of knots $\hat{\mathcal{K}}[3]$ which admits the 2 -cubes action $\kappa$ and is homotopy equivalent to $\mathcal{K}$. First, if $A \in S O_{2},\left(L_{1}, L_{2}, \cdots, L_{n}\right) \in \mathcal{C}_{2}(n),\left(f_{1}, f_{2}, \cdots, f_{n}\right) \in \hat{\mathcal{K}}^{n}$ then

$$
\begin{aligned}
A \cdot \kappa & \left(\left(C_{1}, C_{2}, \cdots, C_{n}\right),\left(g_{1}, g_{2}, \cdots, g_{n}\right)\right)=\left(I d_{\mathbb{R}} \times A\right) \circ\left(C_{\sigma(1)}^{\pi} \cdot g_{\sigma(1)} \circ \cdots \circ C_{\sigma(n)}^{\pi} \cdot g_{\sigma(n)}\right) \circ\left(I d_{\mathbb{R}} \times A^{-1}\right) \\
& =\left(I d_{\mathbb{R}} \times A\right) \circ C_{\sigma(1)}^{\pi} \cdot g_{\sigma(1)} \circ\left(I d_{\mathbb{R}} \times A^{-1}\right) \circ \cdots \circ\left(I d_{\mathbb{R}} \times A\right) \circ C_{\sigma(n)}^{\pi} \cdot g_{\sigma(n)} \circ\left(I d_{\mathbb{R}} \times A^{-1}\right) \\
& =C_{\sigma(1)}^{\pi} \cdot\left(\left(I d_{\mathbb{R}} \times A\right) \circ g_{\sigma(1)} \circ\left(I d_{\mathbb{R}} \times A^{-1}\right)\right) \circ \cdots \circ C_{\sigma(n)}^{\pi} \cdot\left(\left(I d_{\mathbb{R}} \times A\right) \circ g_{\sigma(n)} \circ\left(I d_{\mathbb{R}} \times A^{-1}\right)\right)
\end{aligned}
$$


Topology of knot spaces in dimension 3

$$
=C_{\sigma(1)}^{\pi} \cdot\left(A \cdot g_{\sigma(1)}\right) \circ \cdots \circ C_{\sigma(n)}^{\pi} \cdot\left(A \cdot g_{\sigma(n)}\right)=\kappa\left(\left(C_{1}, C_{2}, \cdots, C_{n}\right), A \cdot\left(g_{1}, g_{2}, \cdots, g_{n}\right)\right)
$$

In the above sequence of equations, the $3 \mathrm{rd}$ equality is perhaps the only step where a little explanation is required.

$$
\left(I d_{\mathbb{R}} \times A\right) \circ C_{\sigma(i)}^{\pi} \cdot g_{\sigma(i)} \circ\left(I d_{\mathbb{R}} \times A^{-1}\right)=C_{\sigma(i)}^{\pi} \cdot\left(\left(I d_{\mathbb{R}} \times A\right) \circ g_{\sigma(i)} \circ\left(I d_{\mathbb{R}} \times A^{-1}\right)\right)
$$

Since both $C_{\sigma(i)}^{\pi}$ and $I d_{\mathbb{R}} \times A$ act on the knot space as a product action, $C_{\sigma(i)}^{\pi}$ acting only in the direction of long axis (identity in the orthogonal directions), while $I d_{\mathbb{R}} \times A$ is by design the identity on the long axis, they commute.

To justify the equivariance with respect to inversion is essentially the same computation. If $C_{i}$ is a 2 -cube, denote $C_{i}$ conjugated by the map $(x, y) \longmapsto(-x, y)$ by $C_{i}^{\prime}$.

$$
\begin{aligned}
I & \cdot \kappa\left(\left(C_{1}, C_{2}, \cdots, C_{n}\right),\left(g_{1}, g_{2}, \cdots, g_{n}\right)\right)=\kappa\left(\left(C_{1}^{\prime}, \cdots, C_{n}^{\prime}\right),\left(I \cdot g_{1}, \cdots, I \cdot g_{n}\right)\right) \\
& =\kappa\left(\left(C_{\iota(1)}^{\prime}, \cdots, C_{\iota(n)}^{\prime}\right),\left(I \cdot g_{\iota(1)}, \cdots, I \cdot g_{\iota(n)}\right)\right) .
\end{aligned}
$$

The first step is argued similarly to the previous case of the $\mathrm{SO}_{2}$-action, the second step is the symmetry axiom of a cubes action.

Note in Proposition 4.3, the choice of involution $\sigma$ is irrelevant, since any two choices differ by an element of $\Sigma_{f}$.

Proposition 4.4 ( $O_{2}$-action on a hyperbolically spliced knot) If $f=J \bowtie L$ where $L$ is a hyperbolic KGL:

- The homotopy equivalence given in Theorem 3.8

$$
S O_{2} \times\left(\left(\prod_{i=1}^{n} \mathcal{K}_{J_{i}}\right) \times_{A_{f}} L_{0}\right) \rightarrow \mathcal{K}_{f}
$$

can be made to be $\mathrm{SO}_{2}$-equivariant, where the $\mathrm{SO}_{2}$ action on $\mathrm{SO}_{2} \times\left(\left(\prod_{i=1}^{n} \mathcal{K}_{J_{i}}\right) \times_{A_{f}} L_{0}\right)$ is a product action, $\mathrm{SO}_{2} \times \mathrm{SO}_{2} \rightarrow \mathrm{SO}_{2}$ is left multiplication and the $\mathrm{SO}_{2}$-action on $\left(\prod_{i=1}^{n} \mathcal{K}_{J_{i}}\right) \times_{A_{f}} L_{0}$ fixes all points.

- $I\left(\mathcal{K}_{f}\right)=\mathcal{K}_{f}$ if and only if there is an isotopy from $L$ to $I(L)$ as unoriented links satisfying:

- The isotopy is orientation-preserving between $L_{0}$ and $I\left(L_{0}\right)$.

- Given the above isotopy, define a signed permutation $\iota \in \Sigma_{n}^{+}$so that $\iota(i)=\epsilon j$ where $\epsilon \in\{ \pm 1\}$ provided the isotopy sends $L_{i}$ to $\epsilon(i) \cdot L_{j}$ where here $1 \cdot L_{j}=L_{j}$ and $-1 \cdot L_{j}$ is $L_{j}$ with orientation reversed.

$-\iota \cdot\left(\mathcal{K}_{J_{1}} \times \mathcal{K}_{J_{2}} \times \cdots \times \mathcal{K}_{J_{n}}\right)=\mathcal{K}_{J_{1}} \times \mathcal{K}_{J_{2}} \times \cdots \times \mathcal{K}_{J_{n}}$.

In this case, $I$ is conjugate (via our homotopy-equivalence) to the self-map of $\mathrm{SO}_{2} \times$ $\left(\left(\prod_{i=1}^{n} \mathcal{K}_{J_{i}}\right) \times_{A_{f}} L_{0}\right)$ given by:

$$
\left(A,\left(J_{1}, J_{2}, \cdots, J_{n}\right), x\right) \longmapsto\left(c(A), \iota \cdot\left(J_{1}, J_{2}, \cdots, J_{n}\right), i(x)\right)
$$

where $c: \mathrm{SO}_{2} \rightarrow \mathrm{SO}_{2}$ and $i: L_{0} \rightarrow L_{0}$ are any orientation-reversing involutions. 
Proof A knot is invertible if and only if $I\left(\mathcal{K}_{f}\right)=\mathcal{K}_{f}$, or equivalently, if and only if $C$ admits an orientation-preserving diffeomorphism $g: C \rightarrow C$ which reverses the longitudinal orientation of $f$. Such a diffeomorphism can be isotoped to preserve the JSJ-decomposition of $C$. Thus $g$ restricts to a diffeomorphism of $V$ and a diffeomorphism of $\sqcup_{i=1}^{n} C_{J_{i}}$, defining a signed permutation $\iota \in \Sigma_{n}^{+}$by how it permutes components and longitudinal orientations of $\sqcup_{i=1}^{n} C_{J_{i}}$. The automorphism of $V$ acts as an involution (by conjugation) on $\tilde{A}_{f}$. This action is negation, since it restricts to negation on the meridional Dehn Twist.

The homotopy equivalence

$$
S O_{2} \times\left(\left(\prod_{i=1}^{n} \mathcal{K}_{J_{i}}\right) \times_{A_{f}} L_{0}\right) \rightarrow \mathcal{K}_{f}
$$

can be made $\mathrm{SO}_{2}$-equivariant via a rather small observation: Restrict the above homotopy equivalence to a map a point in $S O_{2}$, this gives a map $F:\left(\prod_{i=1}^{n} \mathcal{K}_{J_{i}}\right) \times_{A_{f}} L_{0} \rightarrow \mathcal{K}_{f}$. Since $S O_{2}$ acts on $\mathcal{K}_{f}$ we get a new map $S O_{2} \times\left(\left(\prod_{i=1}^{n} \mathcal{K}_{J_{i}}\right) \times_{A_{f}} L_{0}\right) \rightarrow \mathcal{K}_{f}$ by sending $(A, x)$ to $A \cdot F(x)$. This map is also a homotopy-equivalence, since $\tilde{A}_{f}$ splits as a direct sum of two infinite cyclic groups, with one of the generators a meridional Dehn twist.

It seems likely that in the case of an invertible knot, the homotopy equivalence in Proposition 4.4 could be made $\mathrm{O}_{2}$-equivariant for a suitable action of $\mathrm{O}_{2}$ on $S O_{2} \times\left(\prod_{i=1}^{n} \mathcal{K}_{J_{i}}\right) \times_{A_{f}} L_{0}$, although a few computations with rather simple knots such as the Whitehead double of a non-invertible hyperbolic knot demonstrates that with the specific action given in Proposition 4.4 does not suffice [20]. Finding an $\mathrm{O}_{2}$-equivariant homotopy equivalence is likely a subtle problem.

\section{$5 \quad$ Examples}

How difficult is it to compute the homotopy type of a component of $\mathcal{K}$ ? The answer depends on what your starting point is. The simplest starting point would be a companionship-tree description of a knot $f$, together with a list of the relevant hyperbolic isometry groups $B_{L}$ and their representations $B_{L} \rightarrow \Sigma_{n}^{+}$. In that case, one can simply read off the homotopy type of $\mathcal{K}_{f}$ from the theorems in this paper. For the sake of completeness, one also requires knowledge of the groups $\operatorname{Isom}_{\mathbb{H}^{3}}\left(S^{3} \backslash L\right)$ to determine invertibility and the homotopy class of the inversion map of the various companion knots to $f$.

If on the other hand one takes the more traditional starting point of a Reidemeister knot-diagram for $f \in \mathcal{K}$, one needs to first compute the companionship tree $G_{f}$. The algorithm of Jaco, Letscher, Rubinstein [34] allows one to compute $G_{f}$. The JLR algorithm recognises the Seifertfibred pieces together with the singular fibre data. The remaining pieces are hyperbolisable. In practise, one frequently can compute the hyperbolic structure and the representation $B_{L} \rightarrow \Sigma_{n}^{+}$ with SnapPea. As of yet, there is no proof that a SnapPea-type procedure [49] will find the hyperbolic structure on a hyperbolisable manifold. There is an algorithm to find the hyperbolic structure. Haken and Hemion have a solution to the word problem in Haken manifolds [48] and Manning has an algorithm to find the hyperbolic structure on a hyperbolisable manifold, provided it comes equipped with a solution to its word problem [28]. In practise this approach seems rather difficult to implement. 
By and large, the computation of the representations $B_{L} \rightarrow \Sigma_{n}^{+}$is most easily handled by SnapPea. It searches for and frequently finds the canonical polyhedral decomposition of a cusped hyperbolic manifold $M$ [11]. The group of hyperbolic isometries of $M$ is precisely the group of combinatorial automorphisms of the canonical polyhedral decomposition.

If one wishes to avoid the use of computer programs, Kouzi and Sakuma show how one can compute the symmetry groups of various families of hyperbolic links in $S^{3}$ [26] by using the $\mathbb{Z}_{2}$-equivariant JSJ-decomposition of the 2 -sheeted branched cover of $S^{3}$, branched over the link. This is frequently quite practical.

If one takes yet a different starting point, it's potentially much easier to 'compute' the homotopy type of $\mathcal{K}$. At present the class of representations $B_{L} \rightarrow \Sigma_{n}^{+}$for hyperbolic KGLs is not understood. But if one had a list of all such representations, the homotopy type of $\mathcal{K}$ could be described without ever mentioning a knot or a link - it would simply be the recursive closure of the total spaces in Propositions 4.2, 4.3 and 4.4, generated by a point space and our prescribed class of representations $B_{L} \rightarrow \Sigma_{n}^{+}$.

We take the first point of view in this section, and explicitly compute the homotopy type of $\mathcal{K}_{f}$.

\section{Some examples:}

- $\mathcal{K}_{f} \simeq\{*\}$ if $f$ is the unknot.

- $\mathcal{K}_{f} \simeq S^{1}$ if $f$ is a non-trivial torus knot. Here $S^{1}$ is represented by the rotations of a fixed torus knot about the long axis.

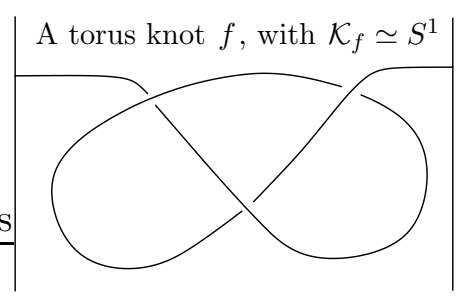

- $\mathcal{K}_{f} \simeq\left(S^{1}\right)^{n}$ if $f$ is an $n$-fold iterated cable of the unknot ie: an $(n-1)$-fold cable of a torus knot. In the figure below, $\mathcal{K}_{f} \simeq\left(S^{1}\right)^{2}$ is represented by the rotations of the knot about the long axis, and the rotation of the cabling parameter respectively $\left(\mathcal{K}_{g}\right.$ in Proposition $4.2)$.

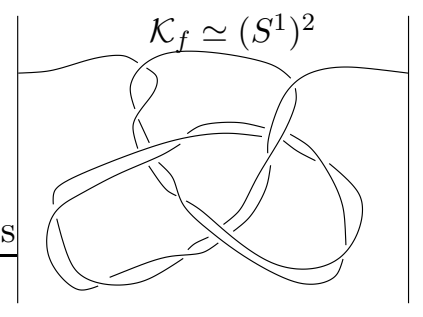

A few of the remaining examples use a signed cycle notation for elements of $\Sigma_{n}^{+}$. The signed cycle $\left(a_{1} a_{2} \cdots a_{k} \pm\right) \in \Sigma_{n}^{+}$is the signed permutation where $a_{1} \longmapsto a_{2}, a_{2} \longmapsto a_{3}$, preserving signs, but $a_{k} \longmapsto \pm a_{1}$, meaning the sign is preserved if $\pm=+$ and reversed if $\pm=-$.

For the Borromean rings $L, B_{L} \simeq \mathbb{Z}_{4}$ where the representation $B_{L} \rightarrow \Sigma_{2}^{+}$sends the generator to the signed 2-cycle (12-), which has order 4 . To those that are curious, the full group of 
isometries of the complement of the Borromean rings has order 48. This includes orientationreversing isometries. The subgroup that preserves the orientation of $S^{3} \backslash L$ has order 24. There is an element of order 3 which cyclically permutes the components of $L$, which can be seen in the 'standard' picture of $L$.

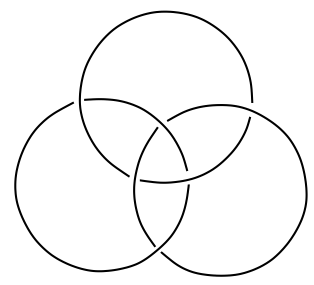

There is an element of order 2 which preserves a component of $L$ but reverses its orientation. This leaves $B_{L}$ as a subgroup of order 4 . This symmetry is perhaps the most difficult to visualize of the symmetries of $S^{3} \backslash L$, though it and the full symmetry group of order 48 can be realized as isometries of $S^{3}$ when $L$ is put in the 'atomic symbol' position.

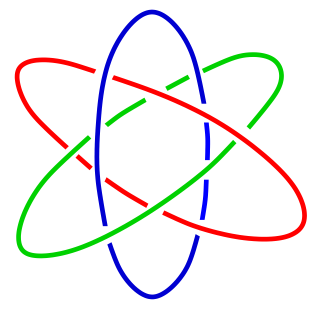

- Consider a 'Borromean sum' of two figure-8 knots, $f=\left(J_{1}, J_{2}\right) \bowtie L$ where $L$ is the Borromean rings, and $\left(J_{1}, J_{2}\right)$ are two copies of the figure- 8 knot then $A_{f}=B_{L}$ and $\mathcal{K}_{f} \simeq S^{1} \times\left(S^{1} \times_{\mathbb{Z}_{4}}\left(\left(S^{1}\right)^{2} \times\left(S^{1}\right)^{2}\right)\right)$. The action of the generator of $\mathbb{Z}_{4}$ on the fibre is induced by the matrix $\left(\begin{array}{cc}0 & -1 \\ 1 & 0\end{array}\right)$, where -1 acts on $S^{1} \times S^{1}$ by rotation by $\pi$ about some fixed point. Thus, $\mathcal{K}_{f}$ is a 6 -dimensional Euclidean manifold.

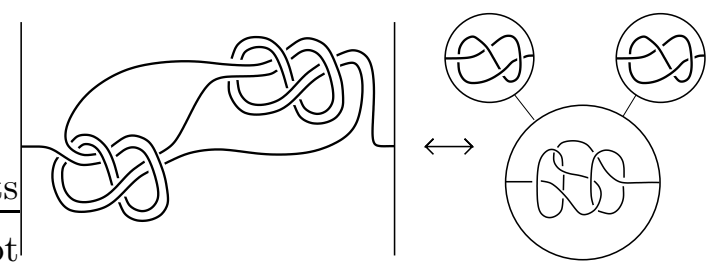

- In the above case, if we replace one of the figure- 8 knots by a trefoil we get $A_{f} \simeq \mathbb{Z}_{2}$ so $\mathcal{K}_{f} \simeq S^{1} \times\left(S^{1} \times_{\mathbb{Z}_{2}}\left(S^{1} \times\left(S^{1}\right)^{2}\right)\right)$. $\mathbb{Z}_{2}$ acts on $S^{1}$ by conjugation, on $\left(S^{1}\right)^{2}$ by rotation by $\pi$ about some fixed point.

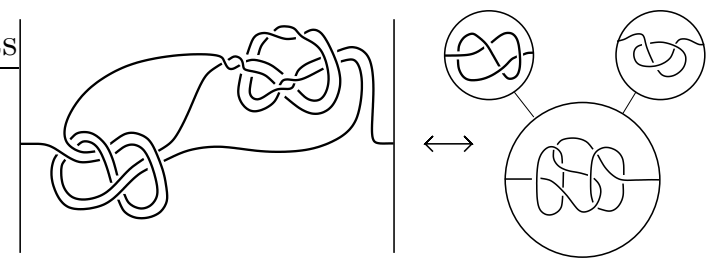

Consider a Whitehead link $L$, then $B_{L}=\mathbb{Z}_{2}$. If $f$ is obtained from $J$ by Whitehead doubling, ie: $f=J \bowtie L$, then: 
- $\mathcal{K}_{f} \simeq S^{1} \times\left(S^{1} \times_{\mathbb{Z}_{2}} \mathcal{K}_{J}\right)$ if $\mathcal{K}_{J}=\mathcal{K}_{I J}$

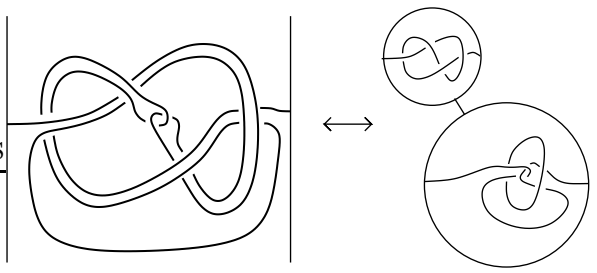

- If $J$ is not invertible, then $A_{f}$ is trivial and $\mathcal{K}_{f} \simeq S^{1} \times S^{1} \times \mathcal{K}_{J}$.

For the knot below, it's a good exercise to compute the homotopy type of $\mathcal{K}_{f}$.

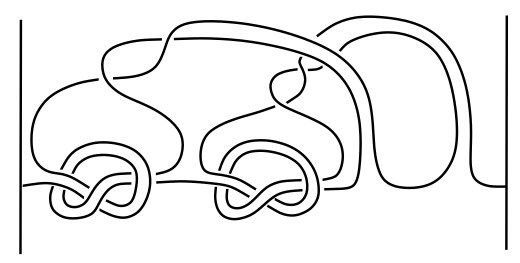

- A more complicated example with interesting homological behavior is given by letting $f_{1} \in$ $\mathcal{K}$ be a trefoil knot. Then $\mathcal{K}_{f_{1}}$ has the homotopy type of a circle. Let $f_{2}$ be the connectsum of 4 copies of $f_{1}, f_{2}=\#_{4} f_{1} \in \mathcal{K}$. Then $\mathcal{K}_{f_{2}}$ has the homotopy type of $\mathcal{C}_{2}(4) \times_{\Sigma_{4}}\left(S^{1}\right)^{4}$. Let $f_{3}$ be a cable of $f_{2}$, then $\mathcal{K}_{f_{3}}$ has the homotopy type of $S^{1} \times\left(\mathcal{C}_{2}(4) \times_{\Sigma_{4}}\left(S^{1}\right)^{4}\right)$. Let $f_{4}$ be the connect-sum of $2^{n}$ copies of $f_{3}$, then $\mathcal{K}_{f_{4}}$ has the homotopy type of $\mathcal{C}_{2}\left(2^{n}\right) \times_{\Sigma_{2}}$ $\left(S^{1} \times\left(\mathcal{C}_{2}(4) \times_{\Sigma_{4}}\left(S^{1}\right)^{4}\right)\right)^{2^{n}}$. In [7] we prove that the homology of $\mathcal{K}_{f_{4}}$ has torsion of order $2^{n+1}$. Moreover, we show for any prime power $p^{n}$, one can find a component of $\mathcal{K}$ whose homology contains torsion of order $p^{n}$.

\section{On the Gramain subgroup}

In Gramain's paper [13] he points out that $\pi_{1} \mathcal{K}_{f}$ contains a group isomorphic to the integers if $f$ is a non-trivial knot. This section investigates that subgroup.

Gramain's subgroup of $\pi_{1} \mathcal{K}_{f}$ is given by the $S O_{2}$-action on $\mathcal{K}_{f}$. Consider the map $S O_{2} \times$ $\mathcal{K}_{f} \rightarrow \mathcal{K}_{f}$ and restrict it to $S O_{2} \times\{f\}$. The induced map $\pi_{1} S O_{2} \rightarrow \pi_{1} \mathcal{K}_{f}$ is the Gramain homomorphism, and the image is Gramain's subgroup. Gramain proved that this map is an embedding provided $f$ is not the unknot. His argument was an application of the BurdeZieschang characterization of the centres of knot groups. We denote the Gramain subgroup of $\pi_{1} \mathcal{K}_{f}$ by $G_{f}$. Sometimes we call generators of this group 'the Gramain element.'

Proposition 6.1 Provided $f$ is a non-trivial knot, the induced map $G_{f} \rightarrow H_{1} \mathcal{K}_{f}$ is an embedding. Moreover, there is a map $\mathcal{K}_{f} \rightarrow \mathrm{SO}_{2}$ such that the composite $\mathrm{SO}_{2} \rightarrow \mathcal{K}_{f} \rightarrow \mathrm{SO}_{2}$ has non-zero degree.

Proof Since we have an inductive description of the homotopy type of the space of long knots, we prove this proposition by induction on the complexity of the JSJ-tree of the knot complement. 
Our base case is a tree with only one vertex - this corresponds to either a torus knot or a hyperbolic knot, in which case $\mathcal{K}_{f} \simeq S^{1}$ or $\mathcal{K}_{f} \simeq S O_{2} \times\left(L_{0} / A_{f}\right)$. In the case of the torus knot our map is simply the homotopy equivalence $\mathcal{K}_{f} \rightarrow S^{1}$. In the hyperbolic satellite case, our map is projection onto the meridional group $\mathcal{K}_{f} \rightarrow \mathrm{SO}_{2}$.

We have three inductive steps,

- $f$ is a cable knot.

- $f$ is a connect-sum.

- $f$ is a hyperbolic satellite.

In the case that $f$ is a cable knot, $\mathcal{K}_{f} \simeq S^{1} \times \mathcal{K}_{g}$. The map out of $\mathcal{K}_{f}$ is projection onto the $S^{1}$ factor.

In the case that $f$ is a connected-sum, $\mathcal{K}_{f} \simeq \mathcal{C}_{2}(n) \times_{\Sigma_{f}} \prod_{i=1}^{n} \mathcal{K}_{f_{i}}$. The map out of $\mathcal{C}_{2}(n) \times_{\Sigma_{f}}$ $\prod_{i=1}^{n} \mathcal{K}_{f_{i}}$ is given by the product of all the maps $\mathcal{K}_{f_{i}} \rightarrow S^{1}$ where we consider $S^{1}$ to be an abelian group.

In the case that $f$ is a hyperbolic satellite knot, we have a fibration a copy of $\mathrm{SO}_{2}$ splits off $\mathcal{K}_{f}$, so we project onto $\mathrm{SO}_{2} \equiv S^{1}$.

Definition 6.2 The image of the generator of $G_{f}$ in $H_{1} \mathcal{K}_{f}$ is called the Gramain cycle, and will be denoted $g_{*}(f)$. The element of $H^{1} \mathcal{K}$ constructed in Proposition 6.1 is called the Gramain co-cycle and is denoted $g^{*}$.

Proposition 6.3 $\left\langle g^{*}, g_{*}(f)\right\rangle= \begin{cases}0 & \text { if } f \text { is the unknot } \\ n & \text { if } f \text { is a connect sum of } n \text { prime knots }\end{cases}$

\section{Questions}

In a paper or Tourtchine [42], the 'first non-trivial 1-co-cycle' detected by the Vassiliev spectral sequence for $\mathcal{K}, v_{3}^{1} \in H^{1} \mathcal{K}$ satisfies:

- $\left\langle v_{3}^{1}, g_{*}(f)\right\rangle=v_{2}(f) \bmod 2$.

- $\left\langle v_{3}^{1},\{a, b\}\right\rangle=0 \bmod 2$, where $\{\cdot, \cdot\}$ is the Poisson bracket on $H_{*} \mathcal{K}$ coming from the cubes action [7].

$g^{*}$ is also trivial on Poisson brackets, so perhaps $v_{2} \cdot g^{*}$ and $v_{3}^{1}$ agree on prime knot components? By Proposition 6.3, they generally do not agree on connect-sums. $v_{3}^{1}$ appears rather mysterious from the point of view of the computations contained in this paper.

An interesting direction to pursue would be to compute the maps $\mathcal{K} \rightarrow A M_{n}$ for $n \geq 3$ coming from the Goodwillie Calculus [6, 39]. As pointed out by Goodwillie and Sinha [40], the map $\mathcal{K} \rightarrow A M_{2}$ is null-homotopic. Since the components of $\mathcal{K}$ are $K(\pi, 1)$ 's, it would be interesting to compute the maps $\pi_{1} \mathcal{K}_{f} \rightarrow \pi_{1} A M_{n} . \pi_{1} A M_{3} \simeq \mathbb{Z}^{2}$, so it's possible that $\pi_{1} A M_{3}$ detects the Gramain element and perhaps one more element, such as projection onto $T_{l} / A_{f}$ in the hyperbolic satellite case. The map $\mathcal{K} \rightarrow \pi_{0} A M_{3} \simeq \mathbb{Z}$ is the universal type- 2 invariant of knots [6], so perhaps $\pi_{1} A M_{3}$ fits better with Tourtchine's $v_{3}^{1}$ cocycle. 
One can give $\mathcal{K}$ the structure of a topological monoid in at least two different ways. One method would be to think of $\mathcal{K}$ as a subspace of $\mathrm{EC}\left(1, D^{2}\right)$ as in [3], then the monoid structure is induced by composition of functions. Another way to endow $\mathcal{K}$ with the structure of a topological monoid is to take the 'Moore loop-space model' for $\mathcal{K}$. Either way, the group completion $\Omega B \mathcal{K}$ of $\mathcal{K}$ is defined $[29,35]$ and has the homotopy type $[3,7]$ of:

$$
\Omega^{2} \Sigma^{2}(\mathcal{P} \sqcup\{*\}) \simeq \Omega^{2} \bigvee_{[f] \in \pi_{0} \mathcal{P}}\left(S^{2} \vee \Sigma^{2} \mathcal{K}_{f}\right)
$$

Since one can construct infinitely many components of $\mathcal{P}$ that have the homotopy type of a product of circles (eg: iterated cable knots), we can conclude that $\Omega B \mathcal{K}$ has $\Omega^{2}\left(\bigvee_{i=2}^{\infty} \bigvee_{\infty} S^{i}\right)$ as a retract. By the results in [7], $\Omega B \mathcal{K}$ does not have the same homotopy type as $\Omega^{2}\left(\bigvee_{i=2}^{\infty} \bigvee_{\infty} S^{i}\right)$ since $H_{*}(\Omega B \mathcal{K} ; \mathbb{Z})$ has torsion of all orders [7] while $H_{*}\left(\Omega^{2}\left(\bigvee_{i=2}^{\infty} \bigvee_{\infty} S^{i}\right) ; \mathbb{Z}\right)$ does not [10].

It may be reasonable to expect that the map $\mathcal{K} \rightarrow A M_{n}$ factors as in the diagram below.

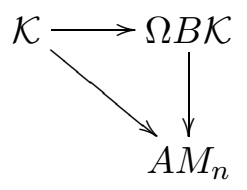

If so, this could be a good platform for studying convergence issues for the Vassiliev spectral sequence, as the group completion map $\mathcal{K} \rightarrow \Omega B \mathcal{K}$ induces an embedding on homology [10], while the fibres of $A M_{n} \rightarrow A M_{n-1}$ are iterated loop spaces on total homotopy fibres of cubical diagrams of Fadell-Neuwirth fibrations [12].

Perhaps the most basic open question related to the homotopy type of $\mathcal{K}$ is the issue of realization of representations $B_{L} \rightarrow \Sigma_{n}^{+}$for hyperbolic KGLs $L$. We have seen a few examples where $B_{L}=\mathbb{Z}_{2}$ or $\mathbb{Z}_{4}$ and a few representations of these groups, but if one spends a little more time considering hyperbolic KGLs, it's clear this is far from all such representations. Alexander Stoimenow has an interesting example $B_{L}=\mathbb{Z}_{n} \rightarrow \Sigma_{n}$ with generator mapping to an $n$-cycle. All the links in the family are hyperbolic since they are alternating, non-split and prime [30].

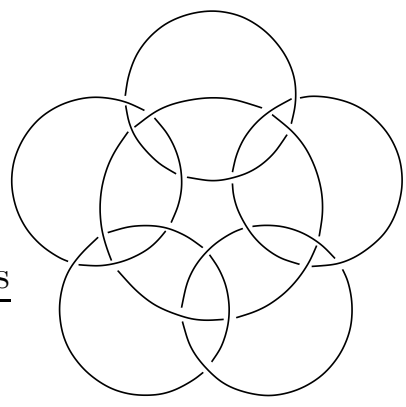

Using the techniques of Kawauchi [25] one can construct many other interesting representations $B_{L} \rightarrow \Sigma_{n}$. Unfortunately, Kawauchi's method can not be used to realise representations $B_{L} \rightarrow$ $\Sigma_{n}^{+}$that are not conjugate to representations into $\Sigma_{n}$ because of some technical restrictions to his imitation theory. Sakuma [37] has another interesting family of examples where $\mathbb{Z}_{2 n}=B_{L} \rightarrow \Sigma_{n}^{+}$ 
is a signed $n$-cycle, ie: the image is generated by $(123 \cdots n-)$, provided $n$ is odd.

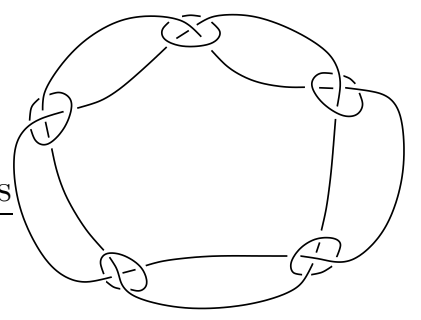

There are of course many further directions one might want to pursue. For a suitable modelspace for $\mathcal{K}, \mathcal{K}$ admits two commuting $\mathrm{SO}_{2}$-actions such that there is a homotopy-equivalence $S O_{2} \backslash\left(S O_{4} \times \mathcal{K}\right) / S O_{2} \simeq \operatorname{Emb}\left(S^{1}, S^{3}\right) / \operatorname{Diff}^{+}\left(S^{1}\right)$. A natural 'next step' would be to determine the $\mathrm{SO}_{2} \times \mathrm{SO}_{2}$ homotopy type of $\mathcal{K}$.

\section{References}

[1] C.M. Boileau, Noeuds rigidement inversibles, Lond. Math. Soc. Lect. Note Ser. 95, 1-18 (1985).

[2] A. Borel, Seminar on transformation groups, Ann. Math. Stud. 46 Princeton University Press, Princeton, NJ (1960).

[3] R. Budney, Little cubes and long knots, Topology 46 No. 1 (2007) 1-28.

[4] R. Budney, JSJ-decompositions of knot and link complements in $S^{3}$, L'Enseignement Mathématique (2) 52 (2006), 319-359.

[5] R. Budney, A family of embedding spaces, Geometry and Topology Monographs 13 (2007).

[6] R. Budney, J. Conant, K. Scannell, D. Sinha, New perspectives on self-linking, Advances in Mathematics. 191 (2005) 78-113.

[7] R. Budney, F. Cohen, On the homology of the space of knots, Geometry and Topology. Vol 13 (2009).

[8] G. Burde, H. Zieschang, Knots. Second edition. de Gruyter Studies in Mathematics, 5. Walter de Gruyter \& Co., Berlin, 2003.

[9] J. Cerf, Topologie de certains espaces de plongements, Bull. S.M.F., tome 89 (1961) 227-380.

[10] F. R. Cohen, T. J. Lada and J. P. May, The homology of iterated loop spaces, Lecture Notes in Math., vol. 533. Springer-Verlag (1976).

[11] D. Epstein, R. Penner, Euclidean decompositions of non-compact hyperbolic manifolds, J. Differential Geom. 27 (1988) 67-80.

[12] T. Goodwillie, M. Weiss, Embeddings from the point of view of immersion theory: Part II, Geom. Topol. 3 (1999), 103-118.

[13] A. Gramain, Sur le groupe fondamental de l'espace des noeuds, Ann. Inst. Fourier. 27 (1977), $29-44$.

[14] A. Hatcher, Homeomorphisms of sufficiently-large $P^{2}$-irreducible 3 -manifolds, Topology. 15 (1976).

[15] A. Hatcher, A proof of the Smale conjecture, Ann. of Math. 177 (1983).

[16] A. Hatcher, Basic Topology of 3-Manifolds, [http://www.math.cornell.edu/ ${ }^{\sim}$ hatcher/3M/3Mdownloads.html].

[17] A. Hatcher, Topological moduli spaces of knots, arXiv [math.GT/9909095]. 
[18] A. Hatcher, Topological moduli spaces of knots, [http://www.math.cornell.edu/ ${ }^{\sim} h a t c h e r /-$ Papers/knotspaces.pdf].

[19] A. Hatcher, D. McCullough, Finiteness of classifying spaces of relative diffeomorphism groups of 3-manifolds, Geometry and Topology, 1 (1997) 91-109.

[20] R. Hartley, Knots and Involutions, Math. Z. 171, (1980) 175-185.

[21] M.W. Hirsch, Differential Topology, Springer-Verlag. (1976).

[22] N.V. Ivanov, Diffeomorphism groups of Waldhausen manifolds, Research in Topology. II. Notes of LOMI scientific seminars, V. 66 (1976), 172-176. J. Soviet Math., V. 12, No. 1 (1979), 115-118.

[23] N.V. Ivanov, Homotopy of spaces of automorphisms of some three-dimensional manifolds, DAN SSSR, V. 244, No. 2 (1979), 274-277. Sovied Mathematics-Doklady, V. 20, No. 1 (1979), 47-50.

[24] W. Jaco, P. Shalen, A new decomposition theorem for 3 -manifolds, Proc. Sympos. Pure Math 32 (1978) 71-84.

[25] A. Kawauchi, Topological imitations, Lectures at Knots 96, ed. Shin'ichi Suzuki (1997) World Scientific. 19-37.

[26] K. Kouzi, M. Sakuma, Symmetry groups of prime knots up to 10 crossings. Knots 90 (Osaka, 1990), 323-340, de Gruyter, Berlin, 1992.

[27] P. Lambrechts, V. Tourtchine, I. Volic, The rational homology of spaces of knots in codimension $>2$, arXiv preprint [math.AT/0703649].

[28] J. Manning, Algorithmic detection and description of hyperbolic structures on closed 3-manifolds with solvable word problem. Geometry and Topology. Vol 6 (2002) 1-26.

[29] J.P. May, The Geometry of Iterated Loop Spaces, Lecture Notes in Mathematics. 271 (1972).

[30] W. Menasco, Closed incompressible surfaces in alternating knot and link complements. Topology 23 (1984), no. 1, 37-44.

[31] J.W. Morgan, H. Bass eds. The Smith Conjecture, Papers presented at the symposium held at columbia University, New York, 1979. Pure and Applied Mathematics, 112. Academic Press.

[32] G. Mostow, Quasiconformal mappings in n-space and the rigidity of hyperbolic space, Pubs. IHES 34 (1968), 53-104.

[33] R. Palais, Local triviality of the restriction map for embeddings, Comment. Math. Helv. 34 (1960) 305-312.

[34] W. Jaco, D. Letscher, H. Rubinstein, Algorithms for essential surfaces in 3-manifold, Contemporary Mathematics, 314 (2002), 107-124.

[35] P. Salvatore, Configuration spaces with summable labels, Cohomological methods in homotopy theory (Bellaterra, 1998), 375-395, Progr. Math., 196, Birkhuser, Basel, 2001.

[36] M. Sakuma, On regular coverings of links, Math. Ann. 260 (1982) 303-315.

[37] M. Sakuma, Uniqueness of symmetries of knots, Math.Z. 192, 225-242 (1986).

[38] K. Scannell, D. Sinha, A one-dimensional embedding complex, Journal of Pure and Applied Algebra 170 (2002), No. 1, 93-107.

[39] D. Sinha, The topology of spaces of knots, preprint. arXiv [math.AT/0202287].

[40] D. Sinha, Operads and knot spaces, J. Amer. Math. Soc. 19 (2006), 461-486.

[41] W. Thurston, Hyperbolic structures on 3-manifolds, I. Deformation of acylindrical manifolds, Ann. of Math. 124 (1986), 203-246.

[42] V. Tourtchine, Calculus of the first non-trivial 1 -cocycle of the space of long knots. preprint arXiv [math.AT/0502518].

[43] V. Tourtchine, On the homology of the spaces of long knots, Independent Moscow University and Université de Paris 7 preprint, May 2001. 
[44] H.F. Trotter, Non-invertible knots exist, Topology 2 (1964), 275-280.

[45] V. Vassiliev, Complements of discriminants of smooth maps: topology and applications, Translations of Mathematical Monographs, 98. American Mathematical Society, Providence, RI, 1992.

[46] I. Volic, Finite-type knot invariants and calculus of functors, Brown University, (2003).

[47] F. Waldhausen, On irreducible 3-manifolds which are sufficiently large, Annals of Math. (2) 103 (1968), 217-314.

[48] F. Waldhausen, Recent results on sufficiently large 3-manifolds, Algebraic and geometric topology (Proc. Sympos. Pure Math., Stanford Univ., Stanford, Calif., 1976), Part 2, pp. 21-38.

[49] J. Weeks, Computation of hyperbolic structures in knot theory, Handbook of Knot Theory. Eds. W. Menasco, M. Thistlethwaite. 2005, Elsevier.

[50] H. Whitney, Differentiable Manifolds, Ann. of Math. 37 (1936) 668-672.

Mathematics and Statistics, University of Victoria

PO BOX 3045 STN CSC, Victoria, B.C., Canada V8W 3P4

Email: rybu@uvic.ca 


$$
0
$$

